This item was submitted to Loughborough's Research Repository by the author.

Items in Figshare are protected by copyright, with all rights reserved, unless otherwise indicated.

\title{
Estimating uncertainty when using transient data in steady-state calculations
}

PLEASE CITE THE PUBLISHED VERSION

http://dx.doi.org/10.1016/j.measurement.2016.07.084

\section{PUBLISHER}

(c) Elsevier

VERSION

AM (Accepted Manuscript)

\section{PUBLISHER STATEMENT}

This work is made available according to the conditions of the Creative Commons Attribution-NonCommercialNoDerivatives 4.0 International (CC BY-NC-ND 4.0) licence. Full details of this licence are available at: https://creativecommons.org/licenses/by-nc-nd/4.0/

\section{LICENCE}

CC BY-NC-ND 4.0

\section{REPOSITORY RECORD}

Buswell, Richard A.. 2019. "Estimating Uncertainty When Using Transient Data in Steady-state Calculations". figshare. https://hdl.handle.net/2134/22245. 


\title{
Estimating uncertainty when using transient data in steady-state calculations
}

\author{
R. A. Buswell ${ }^{1}$ \\ Building Energy Research Group, School of Civil and Building Engineering, Loughborough University, UK
}

\begin{abstract}
When using measurement data for monitoring there is often a desire for steady-state analysis. On-line condition monitoring and fault detection systems are typical applications where the traditional way of treating transient data is to remove it using methods that require tuning using thresholds. This paper suggests an alternative approach where the uncertainty estimate in a particular variable is increased in response to the presence of transients and through propagation, varies the uncertainty in the result accordingly. The formulation of the approach is described and applied to two examples from building HVAC systems. The approach is demonstrated to be a pragmatic tool that can be used to increase the robustness of calculations from time series data.
\end{abstract}

Keywords: Uncertainty, transient data, steady-state detection, condition monitoring, fault detection, HVAC, building energy monitoring

\section{Introduction}

The work described in this paper developed out of the application of condition monitoring and fault detection to heating ventilation and air conditioning (HVAC) Systems in buildings. This has been the focus of research over the last 15-20 years, the aim of which is to help to manage complex systems in some automated sense using data [4, $7,16,9,15,11]$.

The decreasing cost of measurement is increasing the use of smart metering and home energy monitoring systems, where information can be feedback to home owners and also to third party service providers [2]. Additionally, the complexity of building systems needed to cope with onsite generation and energy distribution is all buildings will increase the management of systems [13, 14]. The developments in the built environment are further complicated because is is interconnected through the energy generation and supply systems and so building performance will become increasingly interdependent. Management of these systems will require greater levels of robust automated supervision and since much of the time series analysis in the built environment is based on steady-state calculation methods, having robust means to handle the transient components are important.

Monitoring applications in buildings have fundamental issues of robustness due to unmeasured and typically unidentifiable disturbances, often by the interaction of people. These disturbances combined with the response of the control systems to diurnal variations in ambient conditions result in measurements that will always be transient

Email address: r.a.buswell@lboro.ac.uk (R. A. Buswell) to some extent and hence 'steady-state detection' methods have been developed to filter out transient data $[3,6]$. The algorithms often require threshold selection and can prone to mis-classifying steady-state. This results in a loss of 'good' information and the inclusion of unwanted transient data in the calculation, which tends to generate spurious results and in the case of fault detection, lead to false alarms, which is undesirable.

Figure 1 depicts the problem (taken from [1]). A cooling coil is opened in increments of $10 \%$ of the control signal and left to attain steady-state for about 15 minutes, the goal of which is to characterise it over it's operational range (bottom plot). An algorithm is used to tune the parameters of a steady-state coil model to the data by comparing the predictions of temperature off-the coil (PTSUP) with the measurements (TSUP). The centre plot gives the resultant fit, but attention is drawn to the upper plot. The dots represent the data that are deemed to be in steady-state, using a low-pass filter and gradient threshold method given taken from [10]. The problem is that many steady-state samples are rejected, so much so that the model parameter estimates could be biased. Further 'tuning' only leads to either fewer good points, or the introduction of data from transient region of operation, rather than classifying the data correctly.

Rather than determining rejection criteria, this approach retains all data but scales the uncertainty in the observations according to the detection of transients, thus alleviating the tricky threshold selection and retaining all available information in the data. This uncertainty can then be combined with the other uncertainties and propagated to the result [8]. The technique would typically be applied to each variable under observation and it can be applied in batch format, or recursively for on-line applica- 

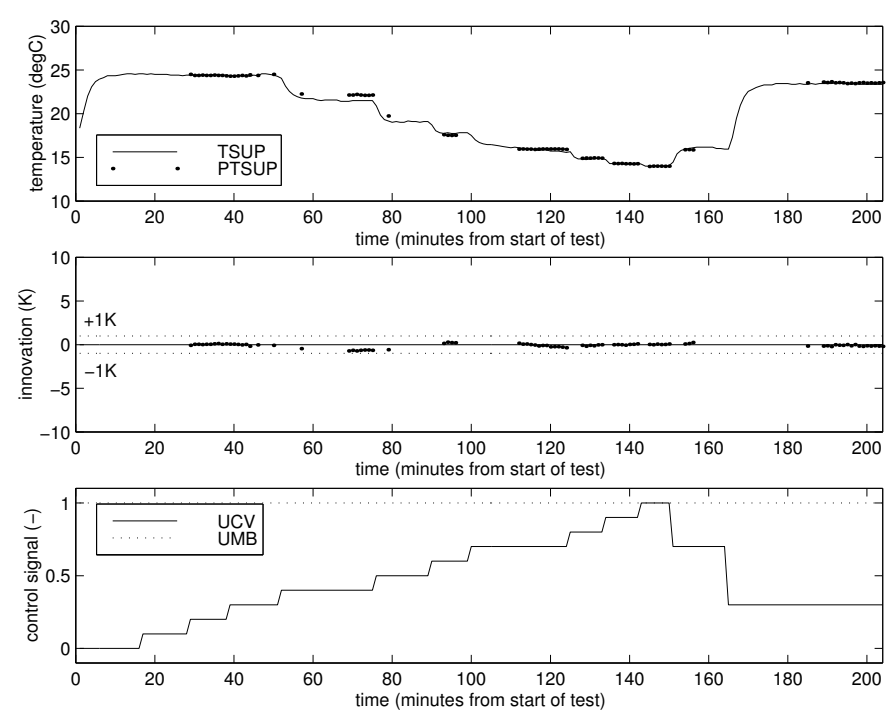

Figure 1: Issues with threshold selection and the reliability of detection of steady-state.

tions. This paper presents the formulation of the method and gives examples of its use in practice.

\section{Method}

The uncertainty in measurements are due to bias in calibration, indeterminable noise, interference from other effects that cannot be eliminated or in the approximation to bulk average properties or quantities. These individual sources are termed elemental uncertainties and are usually quoted at a $95 \%$ confidence interval and combined in quadrature to yield a 95\% confidence level for the measurement,

$$
U_{i}^{2}=U_{a}^{2}+U_{b}^{2}+\ldots+U_{n}^{2},
$$

where $U_{i}$ is the uncertainty in the variable and $U_{a} \rightarrow U_{n}$ are the elemental uncertainties quoted at the $95 \%$ level. Once the variable uncertainties are established, they can be propagated through a particular calculation or analysis using the approach described by Kline and McClintock [8],

$$
U_{y}=\left[\sum_{j=1}^{n}\left(\frac{\partial y}{\partial x_{j}} U_{j}\right)^{2}\right]^{\frac{1}{2}}
$$

Many calculations yield the best results when the system being monitored is stable and close to steady-state, i.e. does not vary with time [7]. Hence, if the data is at or very close to steady-state, then there is negligible uncertainty due to transients in any subsequent calculation. If the system has had an input that drives it towards a new operating condition, then the data will become transient for a period of time, during which any calculation will yield poorer results, because the system is not in steady-state but is 'looking forward' to the new state. In this case, the additional uncertainty in the result of the analysis will be due to the transient nature of the data and hence if accounted for, will yield robust results. Equation 1 can therefore be expanded to become,

$$
U_{i}^{2}=U_{\tau}^{2}+\left(U_{a}^{2}+U_{b}^{2}+\ldots+U_{n}^{2}\right),
$$

where $U_{\tau}$ is the uncertainty due to the transients in the data. The left hand plot in Figure 2 depicts a system that begins in steady-state then at some time $t 1$ there is a step input to the system, such that is drives the variable to a new steady-state, some time later, $t 2$. The variable might respond as shown by the dashed line. If the aim is to calculate a steady-state value from the variable at either the old or new state, then $U_{\tau}=0$ before $t 1, U_{\tau}=$ maximum just after the step input and then $U_{\tau} \rightarrow 0$ as time progresses.

In order to detect the change in state, measure it and evaluate it with respect to the implications on the uncertainty for a calculation, the variable needs to be sampled. The dot-dashed line in the right hand plot of Figure 2 depicts the effects of applying mean and variance calculations to a moving window filter applied to the variable.

Two common methods used to recursivley generate the mean and variance in time series data are: a fixed time window approach which averages consecutive data samples over the length of the window and hence applies an equal weighting to each sample,

$$
\begin{aligned}
\bar{x}_{n} & =\frac{1}{w} \sum_{k=0}^{w} x_{(n-k)}, \\
\sigma_{n} & =\frac{1}{w-1} \sum_{k=0}^{w}\left(\bar{x}_{n}-x_{(n-k)}\right)^{2},
\end{aligned}
$$

where $\bar{x}_{n}$ is the mean of the measurements $x_{(n-k)}$ at sample number $n, w$ is the window length and $\sigma_{n}$ is the sample variance; and an exponentially weighted method that recursively 'forgets' data with each new sample,

$$
\begin{aligned}
\bar{x}_{n} & =\lambda \bar{x}_{n-1}+(1-\lambda) x_{n} \\
\sigma_{n} & =\lambda \sigma_{n-1}^{\cdot}+(1-\lambda)\left(\bar{x}_{n}-x_{n}\right)^{2},
\end{aligned}
$$

where the effective sample mean, $\bar{x}_{n}$ and effective sample variance $\sigma_{n}^{\cdot}$ are calculated. The rate at which the forgetting occurs is controlled by a factor, $\lambda$. The number of samples is represented by the effective number of samples, $n$ and is given by, $n=$ nearest integer $\{\lambda(n-1)+1\}$. Figure 3 shows that practical values for the forgetting factor are in the range $0.8 \leq \lambda \leq 0.967$, corresponding to effective sample sizes of $5 \leq n \leq 30$.

When Equation 4 or 6 are applied to the dotted line in Figure 2, the mean that is generated will in affect bias the measurement of the variable. The approach here is to treat this as the uncertainty in sampling the data, $U_{s m p}$. The variance that is generated by Equations 5 and 7 gives a measure of the magnitude of this. Under steady-state conditions, $U_{s m p}$ is equivalent to what is typically regarded 

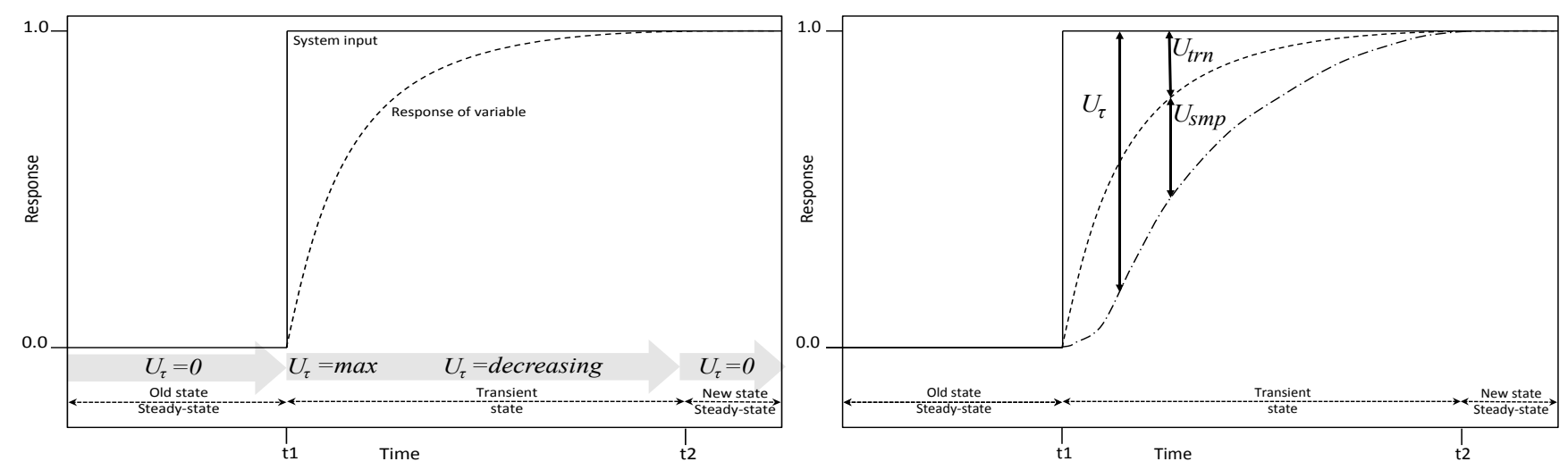

Figure 2: The relationship between the uncertainty due to transients (LHS) and the uncertainty due to sampling the variable with a variance based approach (RHS).
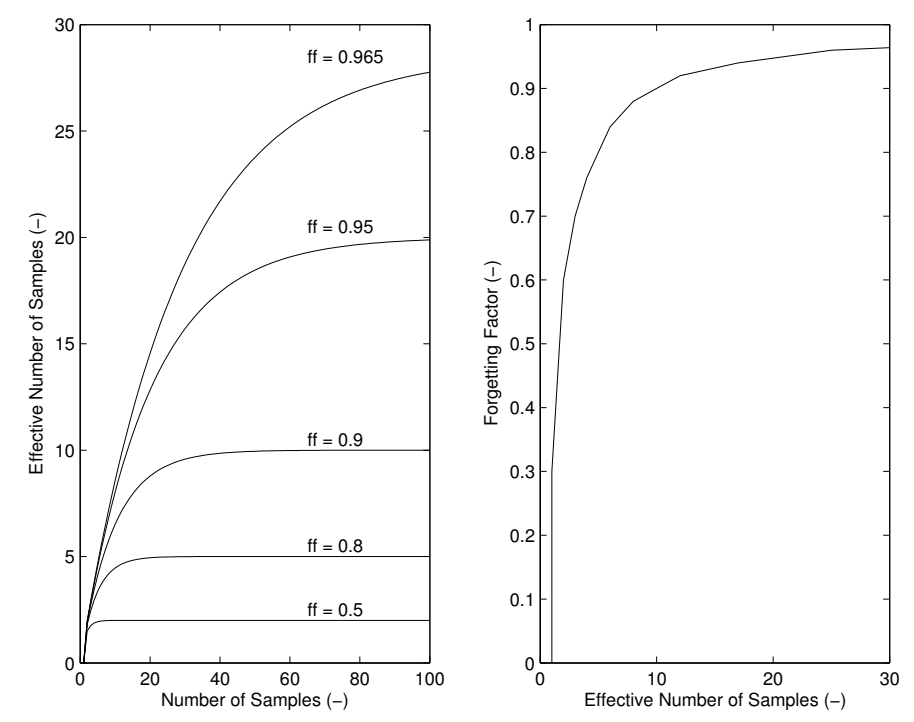

Figure 3: The relationship between effective sample size and the forgetting factor.

as the random uncertainty found in measurements and can be calculated using,

$$
U_{s m p}=\frac{\gamma \sqrt{\sigma_{n}}}{\sqrt{n-1}}
$$

where $\gamma$ is Student's- $t$ statistic, at the $95 \%$ confidence level, $\sigma_{n}$ is the variance and $n$ is the number (or $n$ the effective number) of samples. Under steady-state conditions, the uncertainty derived will be the lowest that can be expected, any increase in variance will indicate transient activity and the uncertainty $U_{\tau}$ will be due to both the sampling and the transient activity, $U_{\tau}=U_{t r n}+U_{s m p}$, illustrated in the right hand plot of Figure 2.

\subsection{Calculating the uncertainty in transient data, $U_{\tau}$}

Most measurements from real systems will have some level of background variation in the data and so even at steady-state $U_{s m p} \neq 0$ and so there will be a minimum level of variance, $\sigma_{n_{\text {min }}}$. The uncertainty can be calculated using Equation 8, since in steady-state $\sigma_{n_{\text {min }}}=\sigma_{n} . \sigma_{n}$ will increase when transients are present and taking the example in Figure 2, will increase to some maximum. The challenge is to estimate the confidence limits at when there is maximum uncertainty $\left(U_{t r n_{t 1}}\right)$ and to then scale this appropriately as the system returns to steady-state $(t 2)$, i.e. when $U_{t r n}=0$, hence $U_{\tau}=U_{s m p}$.

Most real systems will be bounded by practical limits on the expected values of variables and on the expected excitation of the system: for example, if the water flow temperature from a LTHW boiler in the UK is of interest, it might be judged that the maximum likely variation in the temperature measurement is between $10^{\circ} \mathrm{C}$ and $90^{\circ} \mathrm{C}$. In this way the a maximum likely value of uncertainty, associated with a particular variable in a particular application $^{1}, I_{\max }$, can be defined and so $U_{t r n}$ can be described by,

$$
U_{t r n}=I_{\text {max }} f\left(t, \tau, \sigma_{n}\right),
$$

where $f\left(t, \tau, \sigma_{n}\right)$ is a normalised scaling function that creates a time dependant relationship in the decay of $I_{\max }$ to the minimum uncertainty, (i.e. $U_{s m p}$ when $U_{t r n}=0$ ). The rate characteristic rate of decay will also be a function of the system time constant, $\tau$, and the variance, $\sigma_{n}$, is used to observe the system.

\subsubsection{Deriving $f\left(t, \tau, \sigma_{n}\right)$}

The scaling function $f\left(t, \tau, \sigma_{n}\right)$ presented here is based on a step change in a first order system; the characteristics of a measurement $\bar{x}(t)$ generated after a step input, can be described by [12],

$$
\bar{x}(t)=1-e^{-\frac{t}{\tau}} .
$$

\footnotetext{
${ }^{1}$ Such values would usually be judged to be, or calculated at the $95 \%$ confidence level.
} 

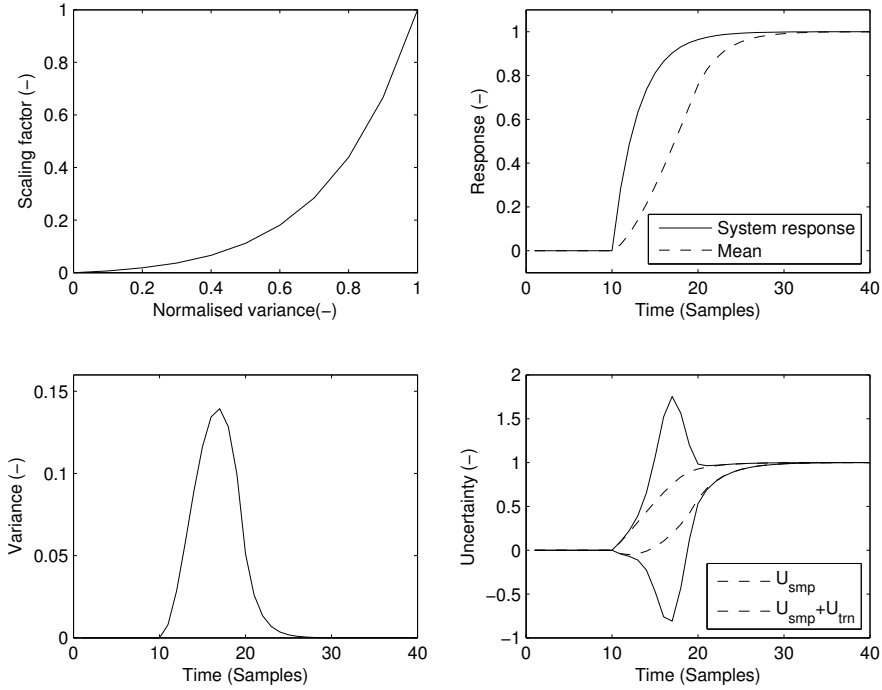

Figure 4: The relationship between $\sigma_{n}^{\prime}$ and the function $f\left(t, \tau, \sigma_{n}\right)$.

Observing Figure 2, it can be seen that the solid line represents the step change, and the dashed line is given by Equation 10 and so $e^{-\frac{t}{\tau}}$, therefore, represents the level of uncertainty between the value and the future value due to the input activity. Steady-state can be considered to exist when the output is at $95 \%$ of its final value [5] and this expression can be normalised to give,

$$
f(t, \tau)=\frac{1}{1-\beta}\left(e^{-\frac{t}{\tau}}-\beta\right)
$$

where $\beta=e^{-\frac{t^{95 \%}}{\tau}}$ and $t^{95 \%}$ is the time after the excitation which the response falls within $5 \%$ of the final value. $\sigma_{n}$ varies between $\sigma_{n_{\min }}$ and $\sigma_{n_{\max }}$ and so can be normalised to give,

$$
\sigma_{n}^{\prime}=\frac{\sigma_{n}-\sigma_{n_{\min }}}{\sigma_{n_{\max }}-\sigma_{n_{\min }}}
$$

It is assumed that $\sigma_{n}$ is a good measure of the transients in the data and so $\sigma_{n}^{\prime} \propto f(t, \tau)$ between the time between the step change occurring and reaching steady-state $(t 1$ and $t 2$ in Figure 2). Since the time constant is defined at the period taken for the output to reach $67 \%$ of its final value [12], $\frac{t}{\tau} \approx 3$ and in order to generate a normalised output, Equation 11 can be modified to yield,

$$
f\left(t, \tau, \sigma_{n}\right)=\sigma_{n}^{\prime} e^{-3\left(1-\sigma_{n}^{\prime}\right)} .
$$

Figure 4 depicts the function given by Equation 13 in the top left had plot. Top right depicts the response of the system where $\tau=3$ and the mean sampled by at 10 sample fixed window. Bottom left shows the resultant variance calculated using Equation 5 and bottom right depicts the $U_{s m p}$ calculated using Equation 8 and $U_{\tau}$ (i.e. $U_{t r n}+$ $\left.U_{s m p}\right)$.

\section{The effect of window size on $U_{\tau}$}

The size of window (or rate of forgetting) is important because it determines the sample size and hence affects the calculation of confidence limits; the greater the number of samples the better. The size of the window also affects the responsiveness of the method to detect changes in the state of the system; the top right hand plot of Figure 4 shows the lag that occurs when calculating the mean. Although it is often desirable to filter the data in this way to avoid odd spikes, or spurious points in the data, its also evident that the shorter the window the more responsive and the longer the window, the less sensitive the method becomes. This means that the selection of the appropriate window length is important when calculating the variance to estimate $U_{\tau}$; as $n$ increases so does $U_{s m p}$ and the maximum variance moves away from the maxim uncertainty and hence becomes less effective as a proxy for measuring $U_{t r n}$.

The following analysis investigates the error generated when averaging functions are employed to evaluate the sample mean and variance in the presence of dynamic effects. The responses generated by a first order system to various forcing functions are used to represent the true values of the measurements. The mean calculated by the filters that sample the true value differ and this difference can be regarded as the total error. The total error and the calculated variance are used to evaluate the performance of the filters. The more desirable filter has a smaller variance and one who's maxima coincides with the peak total error. Three system input characteristics are used here: step, ramp and impulse, given by,

$$
\begin{aligned}
c(t)_{\text {step }} & =1-e^{-t / \tau}, \\
c(t)_{\text {ramp }} & =k^{\prime}\left(t-\tau+\tau e^{-t / \tau}\right), \text { and }, \\
c(t)_{\text {impulse }} & =\frac{1}{\tau} e^{-t / \tau},
\end{aligned}
$$

where $t$ is time (s), $\tau$ is the system time constant (s) and $k^{\prime}$ controls the magnitude of the rate of increase in the ramp input. The number of samples in a given period is given by $n=\frac{t}{I_{n}}$ where $I_{n}$ is the time sample interval (s).

\subsection{Results}

Figure 5 shows the true value, sample response and sample variance for the different system time constants and sample sizes for both the equal and exponentially weighted sampling methods for the first order step input case. On the plot, 'tc' and 'si' refer to 'time constant' and 'sample interval' respectively. The figure shows the lag of the mean in the response to the input which increases as the sample size (window length) increases. The total sample variance increases accordingly. One difference between the two filters is that the exponentially weighted method gives a smaller peak variance at the shorter time constants, whereas when $\tau$ is larger, the equal weighting has a smaller peak variance. 

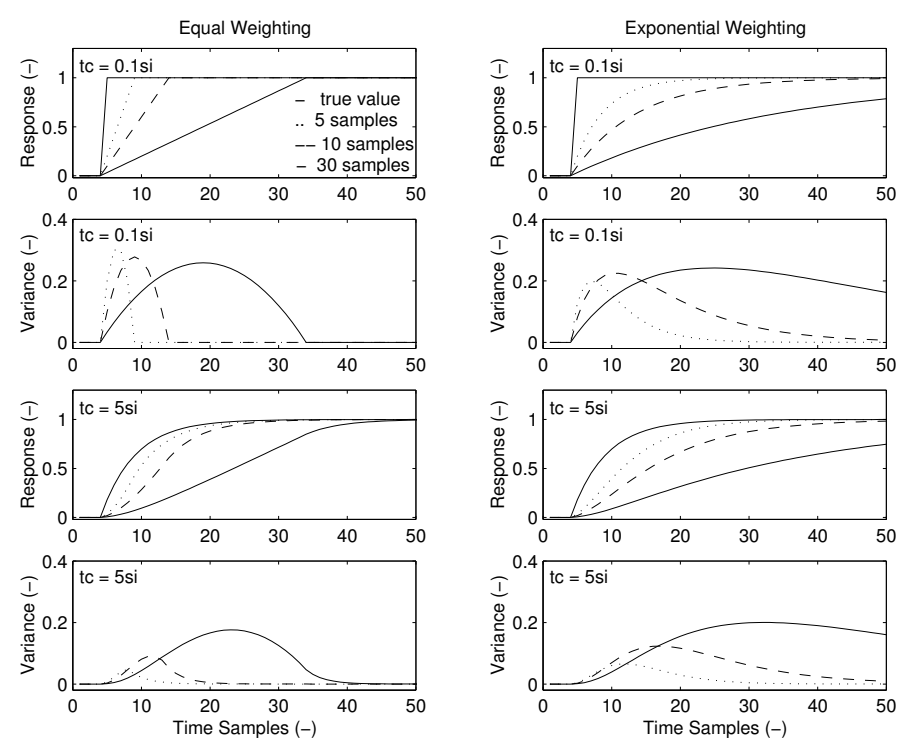

Figure 5: The performance of equally and exponentially weighted averaging methods on the sample uncertainty due to a step input.

Figure 6 demonstrates the coincidence of the total error and variance maxima. There are three observations: the total error associated with the equal weight approach is less than for the exponential method (demonstrated by the areas under the plots); the smaller the sample size, the greater the coincidence of the peak values; and the larger the system time constant is in relation to the sample size, the closer the coincidence.

Figure 7 shows results for a unit ramp input. The ramp functions have been stopped at an arbitrary point to demonstrate the rate at which uncertainty reduces after the disturbance. The steady-state error for a first order response to a ramp input is given by $k^{\prime} \tau$ where $k^{\prime}=1$ for a unit input. After the initial transients, the equal weight method therefore gives constant variance related to the sample size and ramp gradient. The uncertainty is independent of system time constants after the initial transients.

Figure 8 shows the results when the rate of input is increased to $k^{\prime}=2$. What is significant is the effect of $k^{\prime}$ on the response of the exponentially weighted method; it significantly impacts the rate at which the variance returns to zero.

The performance of the methods sampling the systems where an impulse input is generated is shown in Figure 9. For this case, the exponentially weighted method has a lower peak uncertainty, lower total uncertainty and a quicker initial reduction in uncertainty compared to the equally weighted method when the time constant is small with respect to the sample interval. Although the reduction in uncertainty is initially quicker than for the equal weighting, the exponential method is slower to return to zero. Figure 10 shows the relationship between the total error and the sample variance for both methods. As the
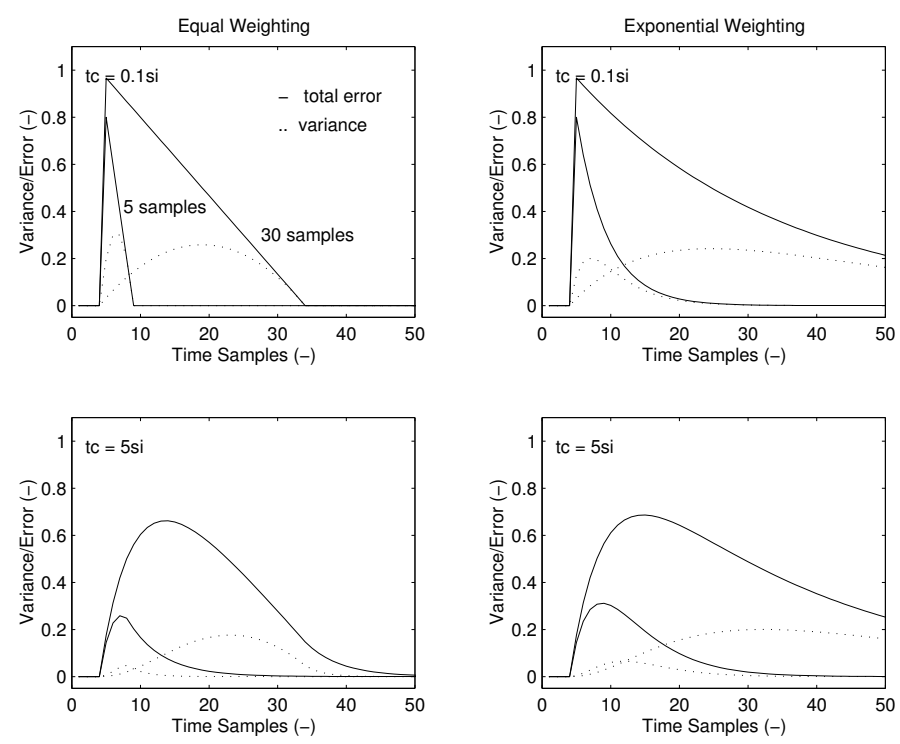

Figure 6: Total error and variance for the equally and exponentially weighted averaging methods in response to a step input.

system time constant increases, the uncertainty becomes less significant. One interesting feature is that due to the rapidity of the system input response and the slower decay of the sampling functions, a sign change in the total error is evident.

\subsection{Summary}

The exponential method considers more samples than the fixed length window method, the 'memory' of the variance, therefore, causes sluggish response to the total error for ramp and step inputs. Conversely, the equal weighting method can generate higher uncertainties when an impulse input is considered, which is particularly apparent when $\tau$ is small. Figure 11 depicts this by plotting the sum of the variance over 50 samples for the step and impulse cases: the ramp and step cases yield similar results relative to the two sampling methods. There is little to distinguish between the filters in terms of the coincidence of the total error with maximum variance. In conclusion, if measurements are taken from a system having predominantly impulse inputs and small time constants, the exponential method will generate less uncertainty. If the system is subject to predominantly ramp and step inputs having longer time constants, as is the case with most typical HVAC systems, the equal weighting method is more appropriate.

\section{Application of the evaluation method for $U_{\tau}$}

The $U_{\tau}$ approach is applied to air temperature measurement in a typical HVAC and a typical domestic setting; both sets of data are from real buildings. The first application demonstrates the variation in $U_{s m p}$ and $U_{t r n}$ and the second the affect of relative contributions to the uncertainty from $U_{\tau}$ and other uncertainties associated 

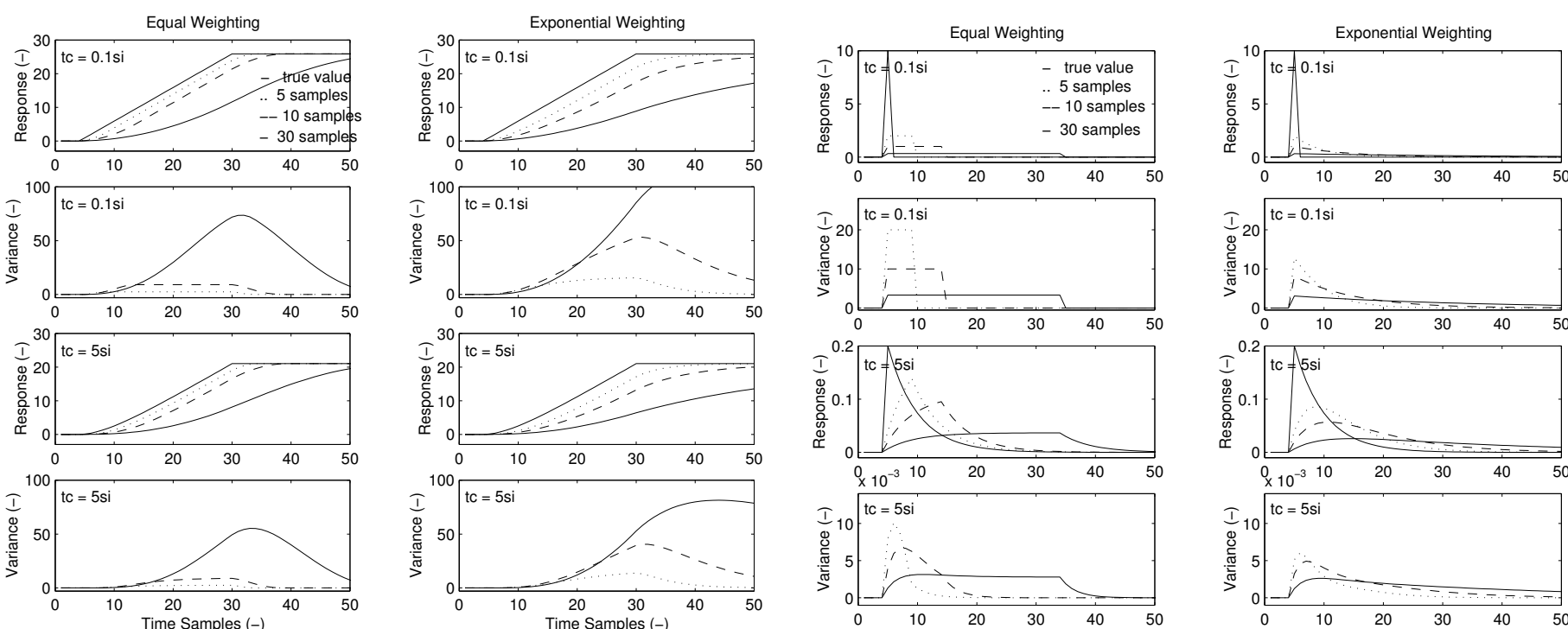

Figure 7: The performance of equally and exponentially weighted averaging methods on the sample uncertainty due to a ramp input; $k^{\prime}=1$, for different time constants.
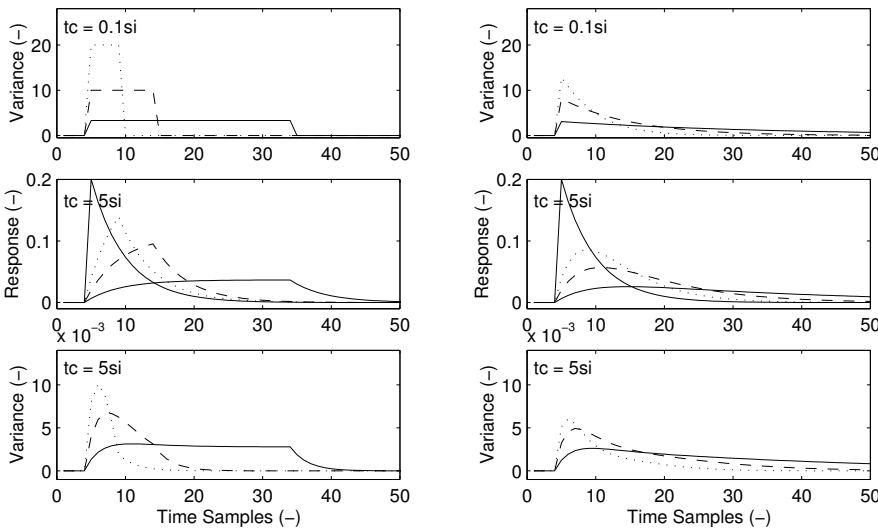

Figure 9: The performance of equally and exponentially weighted averaging methods on the sample uncertainty due to an impulse input.
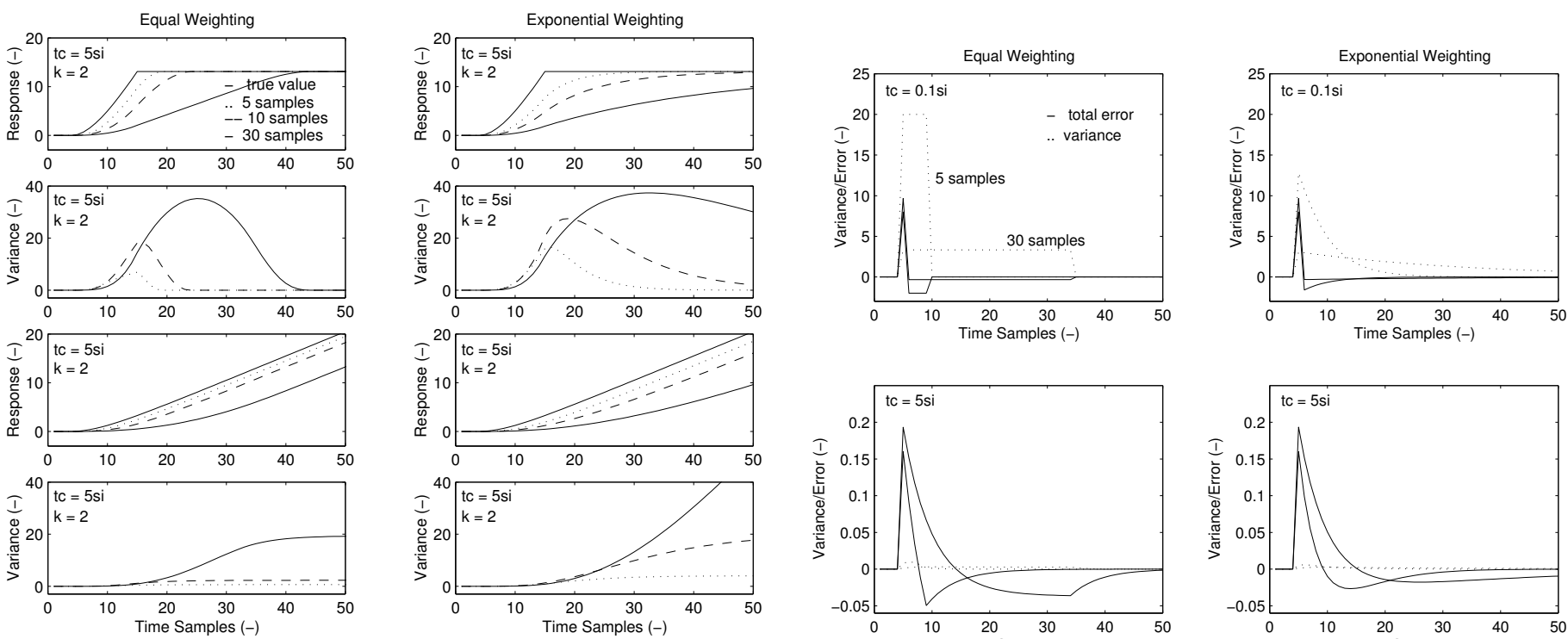

Figure 8: The performance of equally and exponentially weighted averaging methods on the sample uncertainty due to a ramp input; $t c=5 s i$, for $k^{\prime}=1$ and $k^{\prime}=2$.
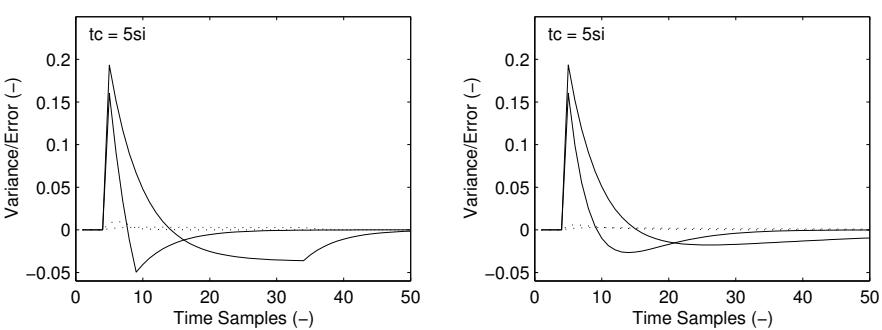

Figure 10: Total error and variance for the equally and exponentially weighted averaging methods in response to an impulse input. 


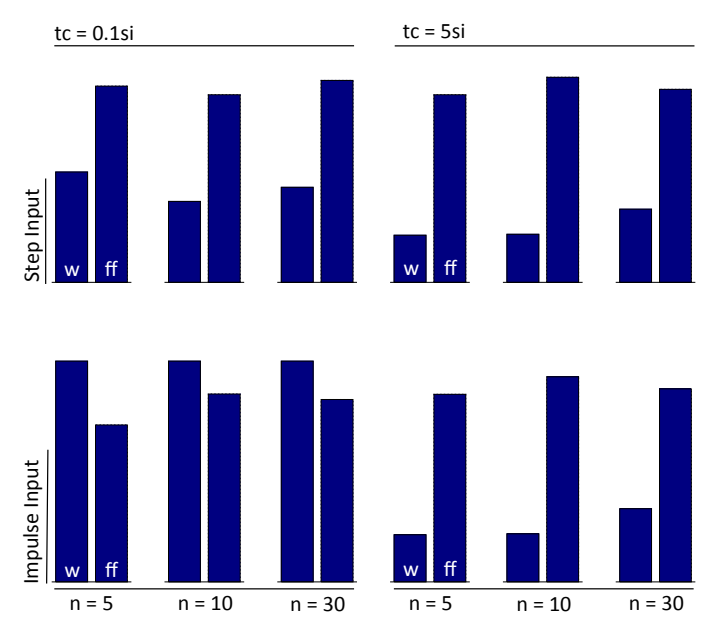

Figure 11: The effects on sample uncertainty due a response to a step and an impulse input for a range of sample sizes $(n)$ and time constants $(t c)$ for equally and exponentially weighted sampling methods, where $s i$ is 'sample interval' and $w$ and $f f$ indicate the fixed window and forgetting factor approaches respectively.

with the measurement. In both cases the equal weighing method has been applied and the window length based on the observed duration of the response of the system to a step input. For example, taking the third plot down on the LHS of Figure 5 , a window length of $\approx 15$ samples would span the response of the system to the input; and would yield a small variance, while remaining responsive to changes. In practice, the method is not overly sensitive to this window length and hence a good estimate can be made by eyeing a sample of data from the system, making the approach practical to implement.

\subsection{HVAC off coil air temperature measurement}

Heating and cooling coils are common components in HVAC systems and are often combined with variable speed fans. Control of the output of coils will be through varying the mass flow rate or temperature of the water flowing through them and hence the temperature of the air off the coil $\left(T_{a o c}\right)$ will be a function of the air and water temperatures into the coil and the air an water flow rates through it. The closeness of $T_{a o c}$ to steady-state will depend on the transients in these inputs due to control action and any unmeasured disturbances.

The $U_{\tau}$ method can be applied to $T_{a o c}$ which is particularly useful in condition monitoring when $T_{a o c}$ could be used to compare against some reference case. Figure 12 depicts data from a real HVAC system where the coil conditions are principally affected by variations in three inputs: the control signal that governs the mass flow rate of chilled water through the coil; the air temperature onto the coil; and the air mass flow rate. The bottom plot shows these normalised against maximum and minimum values
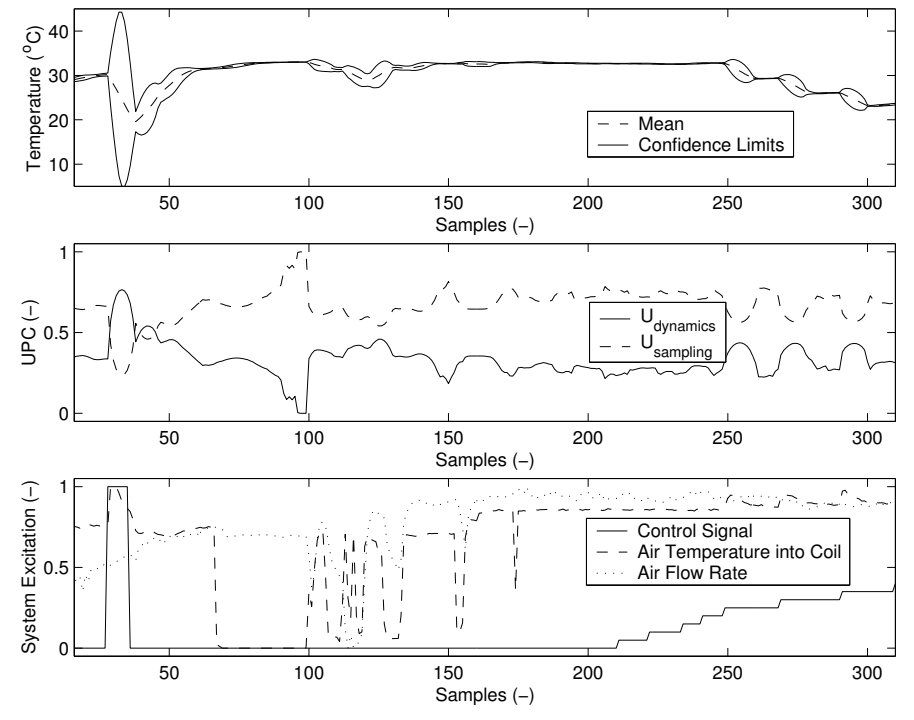

Figure 12: the $U_{\tau}$ method applied to a typical air temperature measurement in an HVAC system.

observed in the data set. The top plot depicts the results from running $T_{a o c}$ through a 10 sample fixed averaging window, calculating the subsequent mean and variance (Equations 4 and 5 ). $\tau \approx 180$ s and the sampling interval is every $60 \mathrm{~s}$. Recorded data was used to establish reasonable estimates of $\sigma_{n_{\min }}=0.07$ and $\sigma_{n_{\max }}=59.8 . U_{\tau}$ was then calculated using Equation 8 and Equation 9 at the $95 \%$ confidence level. The plot shows $\bar{T}_{a o c}$ as the dashed line and $U_{\tau}$ as a solid line. Steady-state can identified and differentiated from when the excitation of the inputs are generating a lack of confidence in the results. Any calculation required using $T_{a o c}$ can now be performed on every sample, propagating the uncertainty through the calculation at each point to improve the robustness of the results. The middle plot shows the balance between $U_{s m p}$ and $U_{t r n}$ as uncertainty percentage contributions $(U P C)$ to $U_{\tau}$. Since other sources of uncertainty have not been considered in this example, these are calculated by, $U P C_{t r n}=U_{t r n} / U_{\tau}$ and $U P C_{s m p}=1-U P C_{t r n}$.

\subsection{Domestic room air temperature measurement}

Monitoring room air temperature in dwellings is increasingly of interest and such measurements are often used as some guide to the comfort levels with in the space or to make some estimation of the energy used to heat or cool the space. Figure 13 shows data from a real UK dwelling during the heating season over a 24 hour period. The temperatures were sampled at 2 minute intervals. The top plot shows the internal air temperature averaged using a 30 sample (60mins) fixed window. The confidence limits are calculated using Equation 3 when $U_{\tau}$ is calculated as described and the calibration uncertainty is $\pm 0.5 \mathrm{~K}$. The bottom plot shows the measured input excitation, being the normalised outside air temperature and the boiler flow 

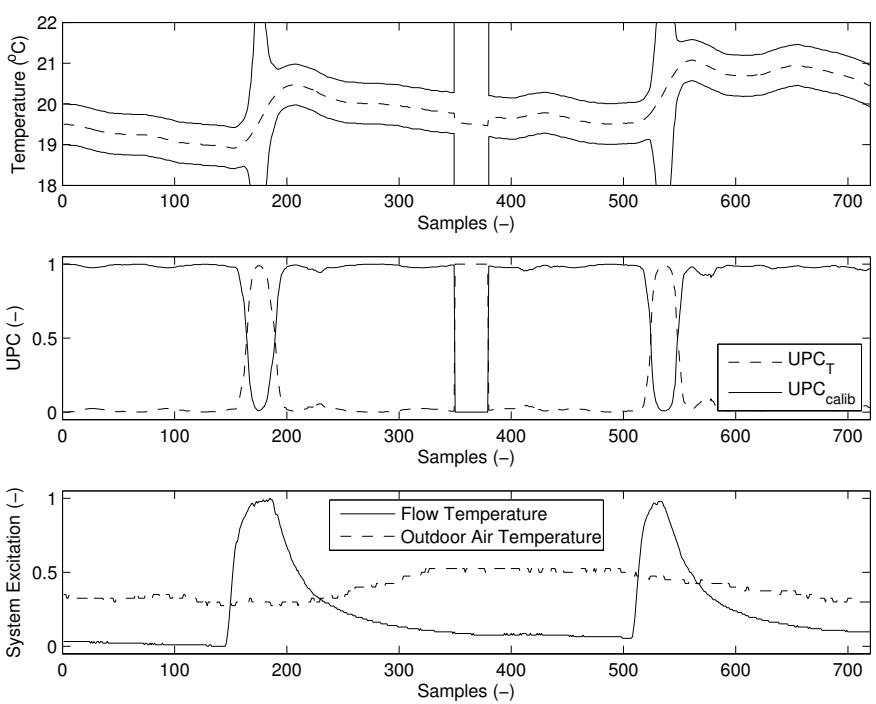

Figure 13: the $U_{\tau}$ method applied to a typical air temperature measurement in a dwelling.

temperature. The boiler flow temperature is used here as a proxy to establish when the system is heating the building: at $\approx 150$ samples, the system switches on, and then turns off at sample $\approx 200$, when the flow pipe goes into free cooling. The system fires once again at sample $\approx 520$. Since the internal air temperature is around $20^{\circ} \mathrm{C}$ and an outside air temperature of $5^{\circ} \mathrm{C}$ would not be uncommon and $I_{\max }$ was judged to be $15 \mathrm{~K}$ : a heat input from an fan heater, or similar might also yield a similar difference.

The UPCs are calculated for $U_{\tau}$ and $U_{\text {calibration }}$ and given on the central plot. As the heating system comes on the building warms up and the $U_{\tau}$ dominates. Once the heating is off, the relatively slow change in outdoor air temperature has little impact and the system approaches steady-state. As the effect of the transient activity drops off the uncertainty in the calibration of the devices dominates. The region of increased uncertainty at samples 350-380 is due to a single sample where the indoor air temperature measurement equaled the outdoor temperature. This was simulated here in order to demonstrate the sensitivity of the approach to unmeasured and unpredictable disturbances (one data point was set to the outside air temperature). For this case, the measurement was in the hall and had the sensor been exposed to a cold gust of air as the door was opened, a dip in the temperature would have been observed. The condition would have been transient and hence we would have less confidence in the results of calculations at this time. By evaluating the uncertainty using the $U_{\tau}$ method, the analysis can be made to be more robust by accounting for the effects of transient data.

\section{Conclusions}

Steady-state calculations are widely used in analysis, but the presence of transients in data can yield spurious results. This is particularly evident in systems that require continual monitoring and that are exposed to unexpected disturbances. Rather than attempt to filter out transient data, this paper presented a robust method of estimating the additional uncertainty in the results due to the use of transient data in steady-state calculations, particularly useful for reducing false alarms in condition monitoring applications.

The theoretical basis for the $U_{\tau}$-method (pronounced 'UT-method') were presented and the sensitivity sample window length investigated for generalised systems, varying time constants and input types. The steps for applying the method are:

1. Establish dominant time constant in the system and likely input type (Step, Ramp, Impulse).

2. Select the averaging method that performs the best:

- if impulse inputs are predominant and the system has small time constants, choose the exponential weighting method (Equations 6 and 7), or,

- if ramp and step inputs are predominant and the system has longer time constants, select the equal weighting method (Equations 4 and 5).

3. Define the maximum likely range that each variable would be expected to encounter in the specific application $\left(I_{\max }\right)$.

4. Apply Equation 9 to each new data point and calculate the uncertainty due to the transients for each variable.

5. Add this $U_{t r n_{k}}$ as an elemental uncertainty to the respective uncertainties in each variable and propagate to the result.

Having originally been developed help generate robust condition monitoring schemes for HVAC system applications, the method now has wider potential application in energy and performance monitoring in the built environment. This is driven through the increasing application of low cost sensing in domestic buildings and the additional complexity of building systems to accommodate renewable energy generation and storage. As buildings become more interconnected to help balance intermittent energy generation and demand, greater levels of automated supervisory monitoring and decision making will be required. Techniques like the $U_{\tau}$-method can contribute to the creation of robust systems.

\section{Acknowledgments}

The author gratefully acknowledges support of the EPSRC funded project, LEEDR: Low effort energy demand reduction, Grant number: EP/I000267/1 and the ASHRAE funded 1020-RP 'Demonstration of fault detection and diagnosis methods in a real building'. 
[1] Buswell, R. A. and J. A. Wright (1998). Practical application of fault detection and diagnosis: Final report on condition monitoring.

[2] DECC (2015). Smart Metering Implementation Programme: Fourth Annual Report on the Roll-out of Smart Meters. Department of Energy and Climate Change, UK.

[3] Dexter, A. and J. Pakenen (2001). Demonstrating Automated Fault Detection and Diagnosis in Real Buildings, Volume VTT Symposium 217. VTT Building Technology, Espoo, Finland.

[4] Glass, A. S., P. Gruber, M. Roos, and J. Tödtli (1994, August). Preliminary evaluation of a qualitative model-based fault detector for a central air-handling unit. In Proceedings of 3rd IEEE Conference on Control Applications, Glasgow, UK.

[5] Gruber, P. (1996). Determination of the tuning parameters of the steadt state detector for a central air handling unit. In IEA Annex 25: Final Report, Volume II - Real time simulation of HVAC systems for building optimisation, Fault deytection and diagnosis, Technical papers of IEA 25, VTT, Finland.

[6] Hyvarinen, J. (1997). IEA Annex 25 Final Report. VTT, Espoo, Finland.

[7] Kim, M., H. Yoon, H, A. Domanski, P, and W. Vance Payne (2008). Design of a steady-state detector for fault detection and diagnosis of a residential air conditioner. International Journal of Refrigeration 31, 790-799.

[8] Kline, S. J. and F. A. McClintock (1953, Jan.). Describing uncertainties in single-sample experiments. Mechanical Engineering 75 No. 3, 3.

[9] Lia, Z., J. J. Paredis, C, G. Augenbroe, and G. Huang (2012). A rule augmented statistical method for air-conditioning system fault detection and diagnostics. Energy and Buildings 54, 154159.

[10] Salsbury, T. I. (1996). Fault Detection and Diagnosis in HVAC Systems using Analytical Models. Loughborough University, UK. $\mathrm{PhD}$ Thesis.

[11] Schein, J., T. Bushby, S, S. Castro, N, and M. House, J (2006). A rule-based fault detection method for air handling units. Energy and Buildings 38, 1485-1492.

[12] Schwarzenbach, J. and K. F. Gill (1992). System Modelling and Control (3rd ed). London: Edward Arnold.

[13] Steijger, L, A., A. Buswell, R, V. Smedley, K. Firth, S, and P. Rowley (2013). Establishing the zero-carbon performance of compact urban dwellings. Journal of Building Performance Simulation 6, 319-334.

[14] Strbac, G. (2008). Demand side management: Benefits and challenges. Energy Policy 36, 4419-4426.

[15] Wang, S., Q. Zhou, and F. Xiao (2010). A system-level fault detection and diagnosis strategy for hvac systems involving sensor faults. Energy and Buildings 42, 477-490.

[16] Wright, J. A. and R. A. Buswell (1999). Demonstration of Fault Detection and Diagnosis in Real Building. Massachusetts Institute of Technology and Loughborough University. 